\title{
3D simulations of device performance for open-shell 3D-Trench electrode detector
}

\author{
Xiaojie Liu ${ }^{1,2,3, a}$, Lipeng Tang ${ }^{1,2,3}$, Chuan Liao ${ }^{1,2,3, b}$, and Zheng $\mathrm{Li}^{1,2,3, \mathrm{C}^{\star}}$ \\ ${ }^{1}$ School of Materials Science and Engineering, Xiangtan University, Xiangtan 411105, China \\ ${ }^{2}$ Center for Semiconductor Particle and photon Imaging Detector Development and Fabrication, \\ Xiangtan University, Xiangtan 411105, China \\ ${ }^{3}$ National - Provincial Laboratory of Special Function Thin Film Materials, and School of Materials \\ Science and Engineering, Xiangtan University, Hunan 411105, China \\ a513529585@qq.com, ${ }^{\mathrm{b}}$ 270883847@qq.com, ${ }^{\mathrm{c}^{*}}$ zhengli58@gmail.com
}

Keywords: electrode silicon detector; Geometric capacitance; Full depletion voltage

Abstract. Therefore silicon semiconductor detectors commonly used as radiation detector. In order to improve charge collection efficiency, an Open-Shell Electrode Detector, namely OSED (Chinese Patent \#ZL201710732524.3) which is based on the Closed Shell-Electrode Detector (CSED), is proposed. The electrical characteristic curves of the cylindrical open shell 3D trench electrode detector, electric potential and electric field distribution, $\mathrm{C}-\mathrm{V}$ curves under different open arc length are discussed and determined in this paper, with is benefit for the design of the detector.

\section{Introduction}

Semiconductor-materials-based detectors are being widely utilized in cutting-edge technologies such as deep space imaging, medical-imaging (e.g., positron emission tomography), In high radiation environment, high radiation-trace detection for the purpose of national security, etc. With high radiation which will cause displacement damage in $\mathrm{Si}$, the defect concentration in the detector will increase. Those defects would affect detector electrical properties such as leakage current increasing, space charge conversing, and charge collection efficiency reducing.

For the improvement of electric field distribution and charge collection, a novel 3D-trench electrode detector has been proposed at BNL in 2009[4], as it shown in Fig.1 [3, 5,6]. Based on the traditonal 3D-Trench Electrode detector and in order to improve charge collection efficiency, an Open-Shell Electrode Detector, namely OSED, is proposed,field and hole (electron) concentration, detector electrical properties such as detector leakage current $(I)$ and capacitance $(C)$ as a function of bias voltage $(V)$ (namely $I-V$ curve and $C$ - $V$ curve), and detector full depletion voltage.

\section{Device description and simulation}

For comparison purposes, a single cell of a non-etch-through square 3D-Trench Electrode detector is shown Figure.1(a). The non-etch-through portion is clearly shown in the bottom of the cell, which typically occupies $6 \%-15 \%$ of the cell volume.

A single cell of a novel open-shell electrode silicon detector with cylinder shape is illustrated in Fig. 1(b). Compared to traditional 3D-trench electrode detector, the electrode of open-shell electrode detector can go though the whole silicon column. The thickness of the detector is defined as $\mathrm{d}$, where $\mathrm{d}=300 \mu \mathrm{m}$. Then open size is defined as $\mathrm{S}$, where $0<\mathrm{s} \leq 10 \mu \mathrm{m}$. When $\mathrm{S}=0$, silicon is not a whole piece, which could fall off. The structure of the detector is determined and all the sizes are given in Fig. 1(b). The central column electrode can be etched all the way through the detector thickness, either from top down or bottom up depending on the specific processing need. The top-etched and bottom-etched trenches are doped the same way (in this case phosphorous or n+-type), and the central column is doped in opposed way (in this case boron or $\mathrm{p}+$-type).The overlapping of the top and bottom trenches 
in the thickness direction ensures the electronic connection between the neighboring cells being minimum to achieving effective cell isolation.[3]
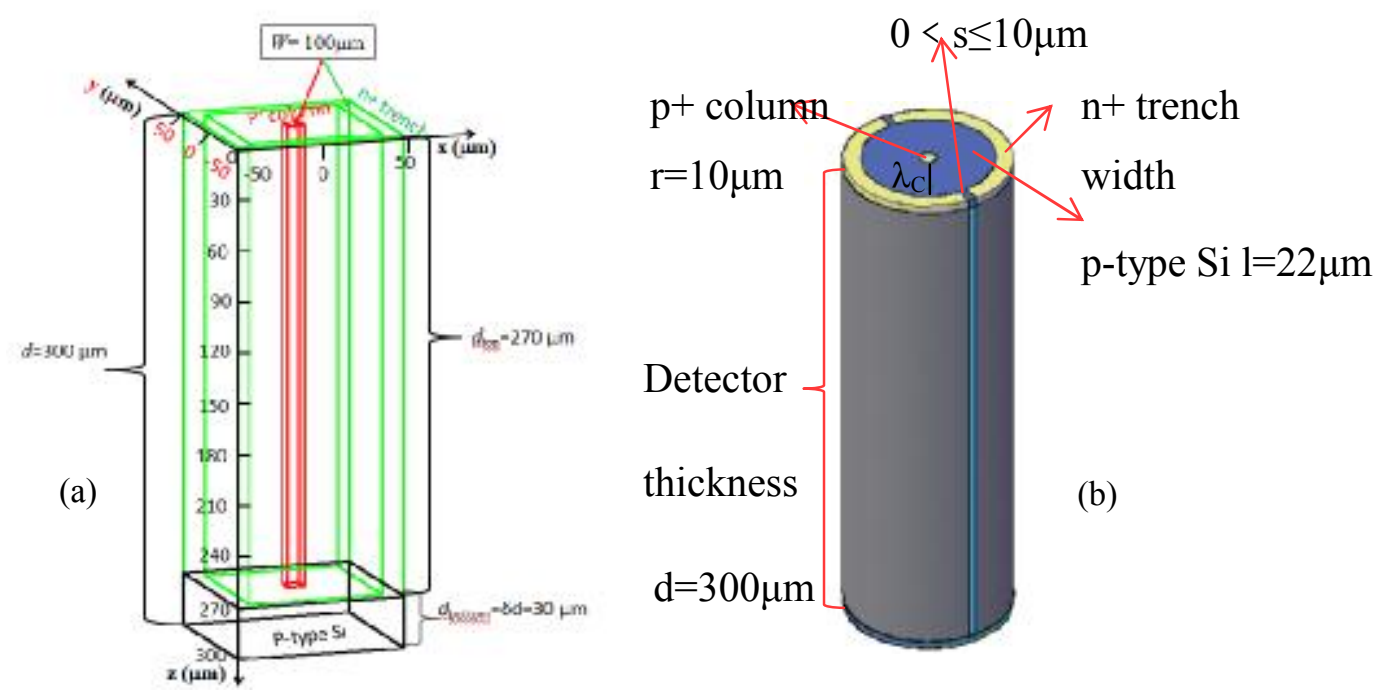

Fig .1 (a)An example of the structure of a square 3D-Trench electrode silicon detector (b)An example of the structure of a square shape novel CSED structure.

\section{Determination of electrode's space}

As a array, which is shown as Fig. $2, \lambda_{C}$ should be controled in a suitable range to save space. As it shown in Fig. The ratio of space area between electrodes to dead space (low electric field space) area, $\lambda_{\mathrm{C}}$, should be controled in a suitable range to save space. As shown in Fig. 2, dead zone area of the array decreases with the decreasing of the rate, and therefore the charge collection efficiency per unit volume can be higher. The rate is defined as follows.

$$
\text { Rate }=\frac{3 \text { - cell gap area }\left(\mathrm{S}_{1}\right)}{\text { triangle area of centers of } 3 \text { units }(\mathrm{S})}
$$

From Fig. 2, it can be included that when radius (R) is in the range of $20 \mu \mathrm{m} \sim 37 \mu \mathrm{m}$, the rate decreases while $\mathrm{R}$ increases. When $\mathrm{R}$ is higher than $37 \mu \mathrm{m}$, the rate changes in proportion to $\mathrm{R}$.The rate is 0 when $\mathrm{R}$ is $37 \mu \mathrm{m}$, and meanwhile dead zone area is the smallest. The $\lambda_{\mathrm{C}}$ is $22 \mu \mathrm{m}$ beside the width of electrode itself. Therefore, the $\lambda_{\mathrm{C}}$ should be $22 \mu \mathrm{m}$.

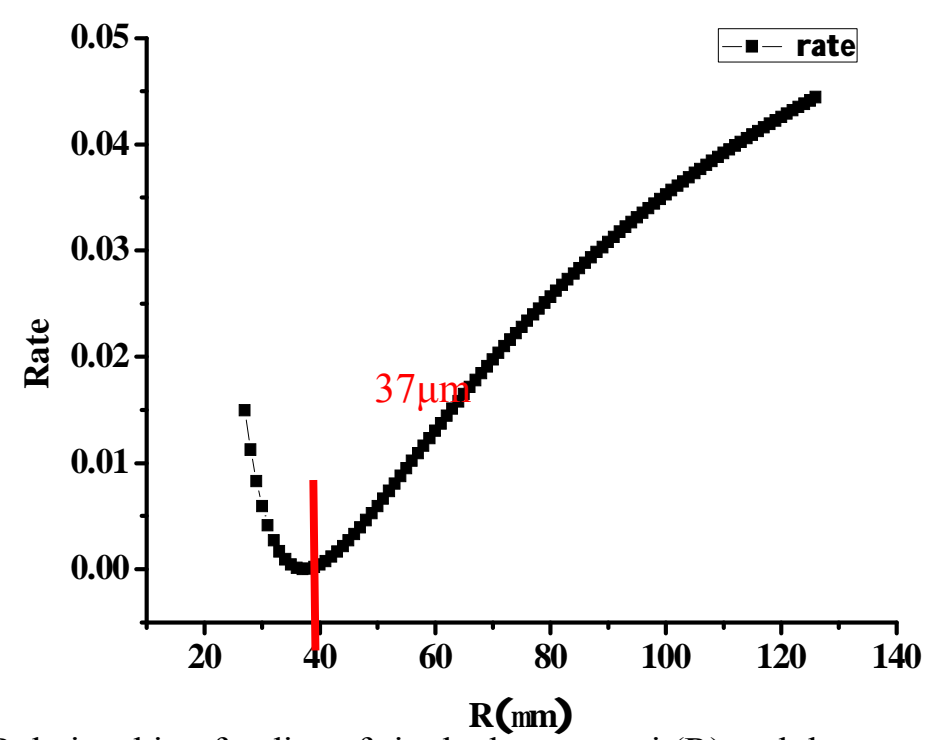

Fig.2 Relationship of radius of single detector unit(R) and the rate 


\section{C-V Characteristics}

For the study of relationship between saturation capacitance of detector and open arc length(S), the $\mathrm{C}-\mathrm{V}$ characteristic curve of detectors with different $\mathrm{S}$ under irradiation flux of $1 \times 10^{16} \mathrm{n}_{\mathrm{eq}} / \mathrm{cm}^{2}$ has been simulated. As it shown in Fig. 3, S can not influence the saturated capacitance. Therefore, it is the process instead of $\mathrm{S}$ should be taken into consideration when designing the detector.

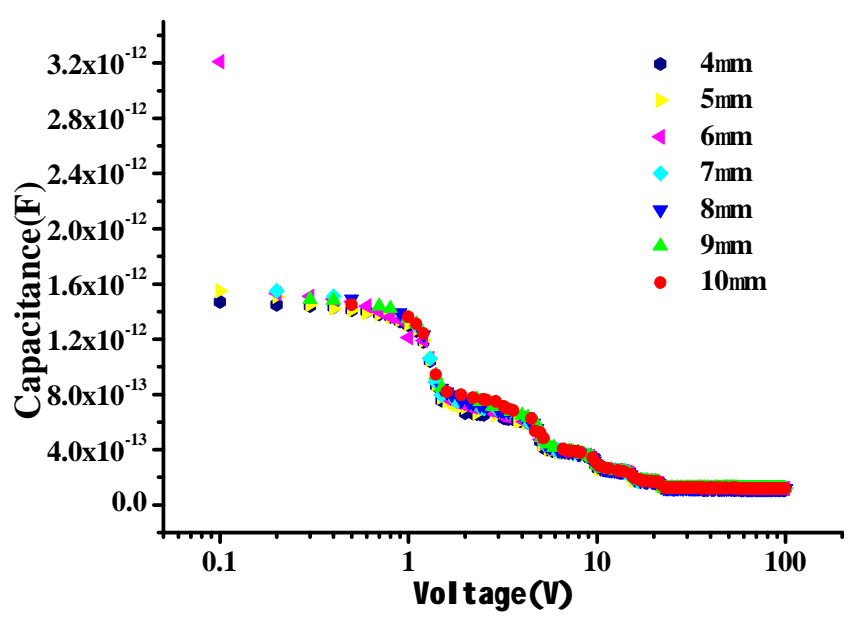

Fig .3 Viriation of capacitance of open-type three-dimensional trench electrode silicon detector with different open arc length under different irradiation intensity

\section{Electric potential}

Fig. 4 shows the potential distribution of open-shell 3D trench electrode silicon detector at $\mathrm{z}=15 \mu \mathrm{m}$ section with $\mathrm{S}$ of $7 \mu \mathrm{m}$ applied bias of $10 \mathrm{~V}$ and no irradiation. In which the vertical axis represents potential, horizonal axis represents position. The figure shows the potential at $\mathrm{z}=15 \mu \mathrm{m}$ section in $3 \mathrm{D}$ form, by which the potential of all the position can be displayed directly. Potential of all position at the section shows a shape of funnel, which is benefit for electronic collection. Electrode in the middle is charge collection electrode. When the electrode is collecting charges, the collected charges are moving downwards alone the funnel, and finally arrives at the bottom of funnel. As it shown in Fig. 4, there is a concave at the top. That is because the concave locates at the opening part, of which the potential is less then that of trench, and that influence little for charge collection. The potential of the concave depends on the size of the opening, the bigger the opening, the potential the lower. Therefore, the opening should not be too large when designing the detector.

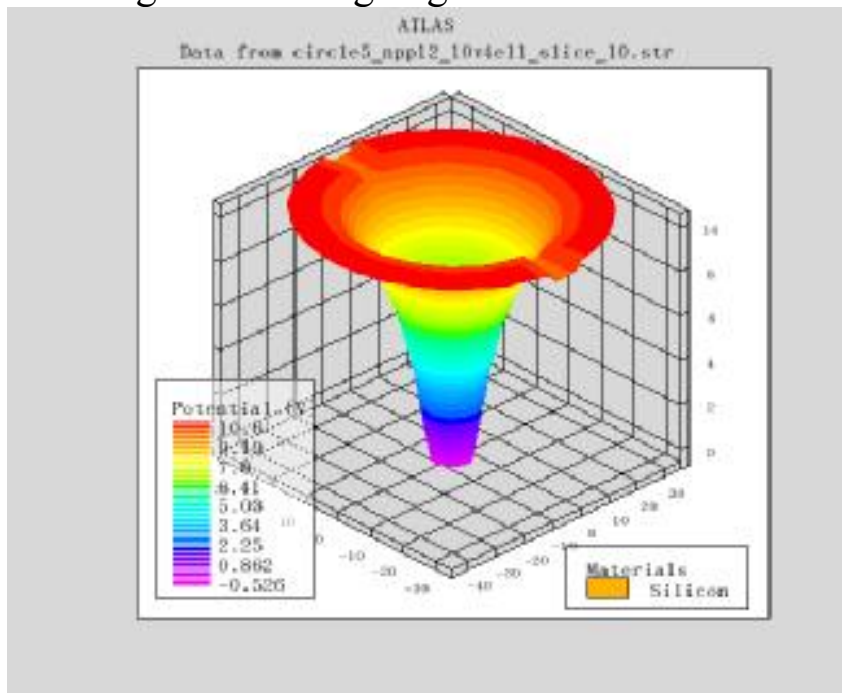

Fig.4 Simulated electric potential profile for the CSED suited here 


\section{Electric field distribution of open-type three-dimensional trench electrode silicon detector}

Fig.5 (a) is electric field distribution of the detector with no irradiation, where $\mathrm{S}$ is $7 \mu \mathrm{m}$ and bias voltage is $10 \mathrm{~V}$. From which it can be included that electric field distributions in electrodes and openings are low, namely the dead zone, in which charges can not be collected. Electric fields of other positions are evenly distributed, and there is no dead zone existed.

(a)

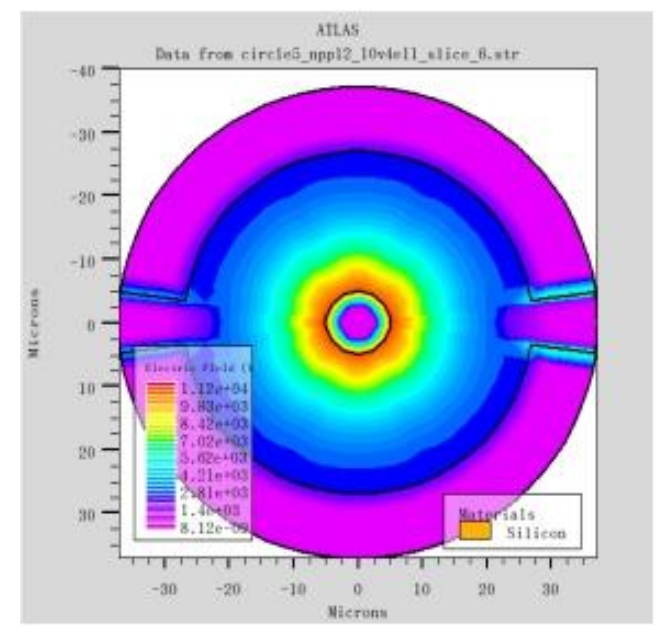

(b)

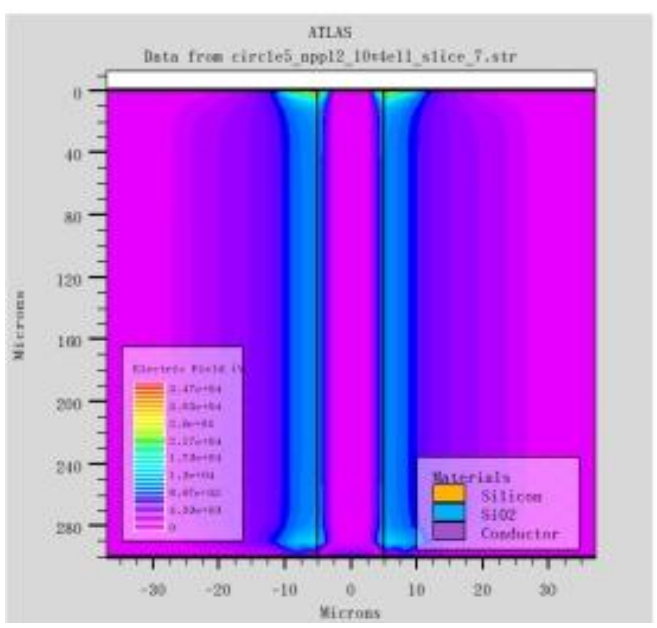

Fig. 5 Electric field distribution of open-type three-dimensional trench electrode silicon detector with no irradiation, where $\mathrm{S}$ is $7 \mu \mathrm{m}$ and bias voltage is $10 \mathrm{~V}$

Based on above analysis, potential of open-type three-dimensional trench electrode silicon detector is evenly distributed.

\section{Conclusions}

A novel Open-Shell Electrode Detector (OSED) has been proposed and described in detail. Full 3D simulations have been conducted by 3D-TCAD on the on the electrical characteristic curves of the cylindrical open shell 3D trench electrode detector (OSED), including, electric potential and electric field distribution, $\mathrm{C}-\mathrm{V}$ curves under different open arc length. Compared to the non-etch-through 3D-Trench electrode detectors, the electric filed profile in the bottom portion (the non-etched region in the non-etch-through 3D-Trench electrode detectors and the bottom trench region in the OSED) is more uniform and much larger in the OSED. The dead space is very small, which is smaller than traditional 3D-trench detector. It requires a double-sided fabrication processing, which is quite standard in 3D detector fabrications.

\section{References}

[1] Bernestein A, Wang Y-F, Gratta G, et al. Nuclear reactor safeguards and monitoring with antineutrino detectors [J]. Journal of Applied Physics, 2002, 91(7: 4672- 4676)

[2] Parker S I, Kenney C J, Segal J. Nuclear Instruments \& Methods in Physics Research, 1997, 395(3):328-343.

[3] Li Z. 3D-TRENCH ELECTRODE DETECTORS: US, US 20120313196 A1[P]. 2012.

[4] Feng M, Li Z, Liu M, et al. Full 3D Simulation of the New Closed Shell-Electrode Detector[C] 2017:09003.

[5] Li Z. Journal of Instrumentation, 2009, 4(03).

[6] Li Z. Nuclear Instruments \& Methods in Physics Research, 2011, 658(1):90-97.

[7] Ding H, Chen J, Li Z, et al. Nuclear Inst \& Methods in Physics Research A, 2015, 796:29-33.

[8] Abe K. 1995. SLAC-E143 collab[J]. Phys Rev Lett, 75: 25 